\title{
Concreto autoadensável ecoeficiente com baixo consumo de cimento
}

\author{
Eco-efficient self-compacting concrete with low cement \\ consumption
}

\section{Lucas da Silva Barboza \\ Izabella Sant'ana Storch (iD \\ Fernando Menezes de Almeida Filho}

Resumo

oncretos autoadensáveis devem apresentar simultaneamente fluidez e coesão, sem a presença de exsudação e segregação. Essas características necessárias do concreto no estado fresco, comumente, só são possíveis mediante altos teores de finos e, por consequência, maior consumo de cimento na produção. A consolidação desse material tem instigado a busca de soluções para reduzir o consumo de cimento e emissões de $\mathrm{CO}_{2}$. Visando a uma contribuição para a sustentabilidade ambiental e a indústria de cimento, esta pesquisa verificou a viabilidade técnica e econômica do concreto autoadensável com baixo consumo de cimento. As densidades dos concretos foram determinadas, assim como sua resistência à compressão e tração por compressão na diametral. Foram analisados três concretos com o consumo de cimento reduzido e um concreto de referência. As relações água/cimento, fíler calcário/cimento e sílica ativa/cimento foram mantidas constantes, enquanto o consumo de cimento variou entre $434,00 \mathrm{~kg} / \mathrm{m}^{3}$ e $325,50 \mathrm{~kg} / \mathrm{m}^{3}$, obtendo-se, assim, redução de até $25 \%$ do consumo de cimento. Os resultados obtidos mostraram que é viável a produção de concretos autoadensáveis com baixo consumo de cimento com garantia da qualidade de suas propriedades mecânicas, com reduções aproximadamente de custos e de emissão de $\mathrm{CO}_{2}$ de $6 \%$ e $19 \%$ respectivamente em relação ao concreto de referência.

Palavras-chave: Baixo consumo de cimento. Concreto autoadensável. Sustentabilidade. $\mathrm{CO}_{2}$.

\section{Abstract}

Self-compacting concretes must simultaneously present fluidity and cohesion without the presence of exudation and segregation. These necessary characteristics of concrete in the fresh state are usually possible only through high contents of fines and, consequently, higher consumption of cement for its production. The consolidation of this material has instigated the search for solutions to reduce cement consumption and $\mathrm{CO}_{2}$ emissions. Aiming to contribute to environmental sustainability and the cement industry, this research studyinvestigated the technical and economic feasibility of selfcompacting concrete with low cement consumption. The densities of the concretes were determined, as well as their resistance to compression and diametral compression traction. The study analysed three concretes with reduced cement consumption and one reference concrete. The water/cement, fibre limestone/cement and active silica/cement ratios were maintained constant, while cement consumption ranged from $434.00 \mathrm{~kg} / \mathrm{m}^{3}$ to 325.50 $\mathrm{kg} / \mathrm{m}^{3}$, thus achieving a reduction of up to $25 \%$ in cement consumption. The results showed that it is feasible to produce self-compacting concrete with low cement consumption, guaranteeing the quality of its mechanical properties, with approximately cost reductions and $\mathrm{CO}_{2}$ emission of $6 \%$ and $19 \%$ respectively in relation to the reference concrete.

Keywords: Low cement consumption. Self-compacting concrete. Sustainability. $\mathrm{CO}_{2}$.
Recebido em 23/01/19 Aceito em 07/09/19 


\section{Introdução}

Com o crescente avanço tecnológico dos materiais e das técnicas da construção civil, misturas de concretos especiais têm surgido na tentativa de superar as deficiências apresentadas pelos concretos convencionais e de atender aos requisitos da sustentabilidade (BARBOZA; ALMEIDA FILHO, 2018).

As características do concreto convencional no estado fresco e endurecido são diferentes das do concreto autoadensável (CAA). O CAA deve apresentar alta fluidez e, simultaneamente, estabilidade da mistura, tendo, assim, propriedades como capacidade de preenchimento de vazios, capacidade de vencer obstáculos e resistência à segregação (PELISSER; VIEIRA; BERNARDIN, 2018). Esse tipo de concreto é uma evolução do concreto convencional de cimento Portland, e seu uso proporciona alta qualidade e durabilidade, o que contribui para a sustentabilidade dos sistemas construtivos (NAJIM; HALL, 2012).

Entretanto, para garantir ao CAA uma fluidez elevada, sem a presença da segregação, grandes quantidades de finos ou de materiais ligantes são empregadas para garantir a qualidade do produto (SUKUMAR; NAGAMANI; RAGHAVAN, 2008). Jagadish, Sudharshan e Ranganath (2003) complementam que o consumo de cimento para concretos autoadensáveis pode chegar à ordem de $450 \mathrm{~kg} / \mathrm{m}^{3}$ a $600 \mathrm{~kg} / \mathrm{m}^{3}$ de concreto.

Diante do contexto industrial, regido pelas elevadas emissões de $\mathrm{CO}_{2}$, oriundas do processo de fabricação do cimento, a produção de concretos mais eficientes com consumo de cimento relativamente reduzido torna-se importante para o avanço do concreto autoadensável, por contribuir também para a redução de impactos ambientais e econômicos (ALI; SAIDUR; HOSSAIN, 2011).

Segundo Mehta e Monteiro (2014), o consumo do concreto tende a crescer a cada ano em âmbito mundial, consequentemente o consumo de cimento também. No entanto, processos e posturas alternativas para a minimização das emissões de dióxido de carbono associadas ao concreto e à produção do cimento devem ser adotados, uma vez que a indústria cimenteira sozinha é responsável por aproximadamente de $5 \%$ a $7 \%$ das emissões globais de $\mathrm{CO}_{2}$ (ALI; SAIDUR; HOSSAIN, 2011; SINDICATO..., 2010; MEHTA; MONTEIRO, 2014).

De acordo com Felix e Possan (2018), na América Latina, o Brasil é considerado um dos países que mais produzem e consomem cimento, tendo chegado a uma produção de 68 milhões de toneladas nos últimos anos. Paralelamente a essa produção, são emitidas na atmosfera toneladas de $\mathrm{CO}_{2}$, uma vez que, para a fabricação de uma tonelada de clínquer, mundialmente são gerados de $700 \mathrm{~kg}$ a $1.100 \mathrm{~kg}$ de $\mathrm{CO}_{2}$ (LIMA, 2010).

É sabido que não é possível aumentar a produção de cimento sem que ocorra o aumento proporcional de emissões de $\mathrm{CO}_{2}$ na atmosfera, pois as estratégias comuns de mitigação são insuficientes. Alguns procedimentos como captura ou até mesmo sequestro do dióxido de carbono são métodos inviáveis no quesito de custo e para contribuir com a sustentabilidade. Portanto, novas estratégias são necessárias para essa contribuição, como a otimização do uso do cimento ou ligantes totais, a substituição parcial do cimento por materiais alternativos, etc. (BARBOZA, 2016).

Entre os trabalhos desenvolvidos que utilizaram composições de concreto autoadensável com adições minerais para reduzir o consumo de cimento com resultados satisfatórios estão Sukumar, Nagamani e Raghavan (2008), que utilizaram cinza volante, consumo de cimento de $250 \mathrm{~kg} / \mathrm{m}^{3}$ e resistência à compressão de 39,6 MPa, Vejmelkova et al. (2011), que utilizaram metacaulim, consumo de cimento de 364 $\mathrm{kg} / \mathrm{m}^{3}$ e resistência à compressão de $67,7 \mathrm{MPa}$, Pelisser, Vieira e Bernardin (2018), que utilizaram a composição de cinza volante e metacaulim, consumo de cimento de $240 \mathrm{~kg} / \mathrm{m}^{3}$ e $28,6 \mathrm{MPa}$, e Barboza e Almeida Filho (2018), que utilizaram sílica ativa e fíler calcário, consumo de cimento de $347,25 \mathrm{~kg} / \mathrm{m}^{3}$ e resistência à compressão de 52,15 $\mathrm{MPa}$.

Com fundamento nesse contexto, o concreto autoadensável com baixo consumo de cimento (CAABCC) pode oferecer vantagens únicas e desempenho mecânico diferenciado, provando que não é necessário alto consumo de cimento para a obtenção de resistência e durabilidade.

Pode-se considerar o CAABCC como uma evolução tecnológica dos concretos tradicionais, oriundo da pesquisa aplicada e resultado da combinação de materiais comuns e de fácil aquisição no mercado da construção civil com dois novos componentes, adições minerais e aditivos químicos, que contribuem para a redução da porosidade e da permeabilidade do concreto (BARBOZA; ALMEIDA FILHO, 2015).

Diversas pesquisas estão sendo desenvolvidas na tentativa de produzir CAA com o consumo de ligantes totais e/ou cimento reduzidos, que garantam as propriedades do concreto no estado fresco e endurecido.

60 Barboza, L. da S.; Storch, I. S.; Almeida Filho, F. M. de 
Long et al. (2017), para um consumo de cimento de 6,2 kg/m³.MPa-1, obtiveram resistência de 24,6 MPa; Singh e Singh (2016), para um consumo de cimento de $13 \mathrm{~kg} / \mathrm{m}^{3} \cdot \mathrm{MPa}-1$, obtiveram resistência de $33 \mathrm{MPa}$; Madandoust e Mousavi (2012), para um consumo de cimento de 9,7 kg/m $\mathrm{m}^{3} . \mathrm{MPa}-1$, obtiveram resistência de $36 \mathrm{MPa}$; Zhao et al. (2015), para um consumo de cimento de 4,9 kg/m³.MPa-1, obtiveram resistência de 56 $\mathrm{MPa}$; e, por fim, Guneyisi, Gesoglu e Ozbay (2010), para um consumo de cimento de 4,8 kg/m³.MPa-1, obtiveram resistência de 37,4 MPa.

Diante desse contexto, dúvidas pertinentes surgem sobre as propriedades mecânicas, a durabilidade e a projeção no futurodesses materiais com consumo de cimento reduzido. Ainda, pode-se complementar que a aplicação do CAABCC em estruturas será alvo de várias pesquisas devido à ausência de vibração e à redução de material cimentício.

Portanto, os aspectos aqui apresentados justificam esta pesquisa, que consistiu em avaliar o efeito da redução do consumo de cimento pela variação do volume de pasta do concreto autoadensável nos requisitos desempenho mecânico e trabalhabilidade.

\section{Indicadores de baixo consumo de cimento}

Tradicionalmente, pode-se expressar a relação entre a massa de cimento utilizada pelo volume de concreto produzido como um fator que mede o consumo de cimento naquela mistura. Em diversos países, entre os quais se inclui o Brasil, é adotado o Sistema Internacional de medida, que costumeiramente é apresentado $\mathrm{em} \mathrm{kg} / \mathrm{m}^{3}$. O método adotado para expressar o consumo não se relaciona com as características dos materiais envolvidos, nem do concreto resultante. Nem sempre um concreto com um consumo de cimento maior estará relacionado com resistência e durabilidade elevadas.

Portanto, pode-se concluir que um melhor indicativo de consumo é aquele que relaciona a quantidade necessária de cimento para produzir certa quantidade de determinado parâmetro, avaliando, assim, o desempenho obtido pela quantidade de cimento empregada. Pode-se expressar esse consumo relativo matematicamente pela Equação 1 (REBMANN, 2011):

$C r=\frac{C}{P}$

Eq. 1

Onde:

C o consumo de cimento; e

$\mathrm{P}$ o parâmetro escolhido.

Um dos principais parâmetros utilizados para retratar a eficiência estrutural do concreto é a resistência à compressão aos 28 dias. Dessa maneira, um índice de consumo pode ser gerado levando-se em consideração a resistência obtida pelo concreto e a quantidade de cimento em um metro cúbico de concreto necessária para se produzir $1 \mathrm{MPa}$ de resistência à compressão. Por exemplo, um concreto que apresenta um consumo de cimento igual a $400 \mathrm{~kg} / \mathrm{m}^{3}$ atingiu uma resistência à compressão de $35 \mathrm{MPa}$ aos 28 dias; assim, esse concreto expressará um $\mathrm{Cr}$ igual a $11,43 \mathrm{~kg} / \mathrm{m}^{3}$ para atingir $1 \mathrm{MPa}$. Não são todas as situações em que o parâmetro da resistência à compressão é essencial; por exemplo, em pisos a resistência de maior interesse é a tração na flexão. Sendo assim, outros índices podem ser propostos, conforme o parâmetro mais importante.

Pode-se também variar esse parâmetro levando-se em consideração os diversos tipos de aglomerantes empregados na mistura, como sílica ativa, cinza volante, escória de alto forno, etc.

Um dos quesitos desta pesquisa é o aspecto ambiental, com o interesse em estimar a quantidade de $\mathrm{CO}_{2}$ emitida por megapascal produzido por meio do consumo de cimento de cada concreto. Esse índice de consumo está relacionado à produção de clínquer principalmente. Algumas características do concreto são consideradas, como o calor de hidratação, porque esse aspecto está ligado diretamente à quantidade de clínquer disponível. Ainda assim, pode-se propor um índice de quilograma de clínquer por megapascal produzido por metro cúbico de concreto. $\mathrm{E}$, por fim, calcular a quantidade de $\mathrm{CO}_{2}$ emitido por megapascal produzido.

\section{Materiais e programa experimental}

Nesta seção é apresentado estudo experimental que engloba o desenvolvimento do concreto autoadensável com baixo consumo de cimento e a investigação experimental para o estudo de suas propriedades no estado fresco e endurecido, visando responder às questões que motivaram este trabalho. Ressalta-se que nesta 
pesquisa considerou-se concreto com baixo consumo de cimento aqueles que apresentam baixa relação consumo-resistência em comparação aos concretos existentes.

Posteriormente, são descritos todos os materiais utilizados nos diversos concretos ensaiados, com suas principais características. Na sequência, apresentam-se os ensaios executados. Os ensaios foram conduzidos no Laboratório de Estruturas e Materiais do Unasp, utilizando-se uma prensa universal servo-hidráulica, classe I. A proposta experimental foi dividida em escolha e caracterização dos materiais constituintes das misturas, método de dosagem e ensaios de caracterização do concreto nos estados frescos e endurecido.

\section{Materiais utilizados}

Foi utilizado apenas um tipo de cimento, cimento Portland composto com pozolana e classe de resistência de $32 \mathrm{MPa}$ (CPII Z 32). Como agregado miúdo natural utilizou-se areia fina quartzosa, com dimensão média característica de $0,3 \mathrm{~mm}$ e módulo de finura igual a 0,82. Como adição adotou-se a sílica ativa, escolhida com o intuito de gerar o refinamento dos poros por meio da açãopozolânica e de empacotamento nos níveis abaixo da granulometria do cimento. A sílica ativa utilizada é oriunda do processo de fabricação das ligas de ferro silício, com diâmetro médio de $0,40 \mu \mathrm{m}$, e possui massa específica de $2,22 \mathrm{~g} / \mathrm{cm}^{3}$. Utilizou-se também um fíler inerte proveniente do calcário, compacto e de granulação fina na coloração branca, chamado comercialmente de pó de calcário, com dimensão máxima característica de $45 \mu \mathrm{m}$ e densidade aparente de aproximadamente $1,2 \mathrm{~g} / \mathrm{cm}^{3}$.

Foi selecionado um único agregado graúdo natural britado de origem basáltica, com dimensão máxima característica de $9,5 \mathrm{~mm}$, módulo de finura de 5,84 e classificado como brita 0 .

Optou-se por utilizar o aditivo Advacast 525 para todos os concretos elaborados, produto à base depolicarboxilatos, com massa específica média de $1,056 \mathrm{~g} / \mathrm{cm}^{3}$.

\section{Dosagem e estado fresco do concreto}

As amostras de concreto foram preparadas com volume de pasta de $40 \%$ no concreto de referência (CAAR), $33 \%$ no $\mathrm{CAABCC} 1,32 \%$ no $\mathrm{CAABCC} 2$ e, por fim, 30\% no $\mathrm{CAABCC} 3$, seguindo as recomendações propostas por Gomes (2002) para a metodologia de dosagem.

As dosagens foram desenvolvidas com base nos limites exigidos para atender à classe de agressividade ambiental do tipo II da NBR 12655 (ABNT, 2015). Esses limites constituem-se de consumo de cimento de no mínimo $280 \mathrm{~kg} / \mathrm{m}^{3}$, relação $\mathrm{a} / \mathrm{c}$ de no máximo 0,60 e resistência à compressão aos 28 dias deno mínimo $30 \mathrm{MPa}$. Esses parâmetros devem ser atendidos simultaneamente. A relação a/c utilizada para todas as misturas foi de 0,40 .

O teor ótimo de aditivo superplastificante foi de 2,00\%, valor obtido por meio do ensaio de Funil Marsh para determinar o tempo de escoamento e do ensaio de miniabatimento para determinar o abatimento segundo a NBR 13276 (ABNT, 2002) para um tempo de $10 \mathrm{~min}$. Foi utilizada pasta de cimento com adição de 10\%, $20 \%, 30 \%$ e $40 \%$ de fíler calcário e de $10 \%$ de sílica ativa, valor usualmente adotado por diversos pesquisadores, como Santos (2012), Almeida Filho (2006), Gomes (2002) e Gettu e Aguillo (2004). Tendo em vista que a mistura com o teor de $40 \%$ de fíler de calcário, maior teor utilizado nesta pesquisa, obteve resultados satisfatórios, com diâmetro de espalhamento no intervalo de $18,0 \pm 1,0 \mathrm{~cm}$ e tempo $\mathrm{T}_{115}$ no intervalo de $3 \pm 1 \mathrm{~s}$, sem a presença de segregação, optou-se por utilizar essa proporção, uma vez que o objetivo principal deste trabalho é a redução do consumo de cimento.

O consumo de materiais para as misturas encontra-se na Tabela 1.

A mistura foi realizada em betoneira de modelo por gravidade, com capacidade do tambor de $400 \mathrm{~L}$, capacidade de mistura de 320 L e rotação do tambor de 28 RPM, seguindo a mesma ordem de mistura para todas as dosagens. Tanto o concretode referência quanto os concretos com consumo de cimento reduzido foram misturados na seguinte sequência: agregado graúdo mais 3/4 da água total de amassamento (água para a pasta e água para saturar os agregados), misturados por $30 \mathrm{~s}$; mais cimento, sílica ativa, o pó de calcário com o superplastificante e o restante da água, misturados por $120 \mathrm{~s}$; e, por fim, o agregado miúdo, misturado por outros $120 \mathrm{~s}$. A mistura ocorreu em sequência, suficiente para promover a homogeneização dos materiais presentes na betoneira, totalizando, assim, aproximadamente um tempo de mistura igual a 4,5min.

62 Barboza, L. da S.; Storch, I. S.; Almeida Filho, F. M. de 
Tabela 1 - Consumo de materiais por metro cúbico de concreto das dosagens analisadas

\begin{tabular}{c|c|c|c|c}
\hline Materiais & CAAR & CAABCC 1 & CAABCC 2 & CAABCC 3 \\
\hline Cimento $\left(\mathrm{kg} / \mathrm{m}^{3}\right)$ & 434,06 & 358,10 & 347,25 & 325,54 \\
Fíler calcário $\left(\mathrm{kg} / \mathrm{m}^{3}\right)$ & 173,62 & 143,24 & 138,90 & 130,22 \\
Sílica ativa $\left(\mathrm{kg} / \mathrm{m}^{3}\right)$ & 43,41 & 35,81 & 34,72 & 32,55 \\
Areia $\left(\mathrm{kg} / \mathrm{m}^{3}\right)$ & 648,70 & 724,39 & 735,20 & 756,82 \\
Brita $\left(\mathrm{kg} / \mathrm{m}^{3}\right)$ & 973,06 & $1.086,56$ & $1.102,80$ & $1.135,23$ \\
$\mathrm{SP}\left(\mathrm{kg} / \mathrm{m}^{3}\right)$ & 8,68 & 14,97 & 15,62 & 14,97 \\
Água $\left(\mathrm{kg} / \mathrm{m}^{3}\right)$ & 173,62 & 143,24 & 138,90 & 130,22 \\
\hline
\end{tabular}

Alcançada a trabalhabilidade esperada da mistura, ela é submetida aos ensaios de estado fresco, slump-flow test, caixa-L, Funil-V e Tubo-U, de acordo com os parâmetros da NBR 15823 (ABNT, 2017). Não se utilizou nos ensaios realizados para caracterização dos concretos frescos qualquer meio de adensamento, além do peso próprio do concreto.

Os concretos produzidos foram colocados em moldes e recobertos por uma lona plástica a fim de evitar a perda da água para o meio. Após $24 \mathrm{~h}$, as amostras foram desmoldadas e submetidas à cura submersa em tanque de água até a data prevista de ensaio: 3, 7, 28 e 91 dias.

\section{Concreto no estado endurecido}

A massa específica no estado endurecido foi determinada de acordo com a NBR 9778 (ABNT, 2009) aos 28 dias, utilizando-se corpos de prova com $150 \mathrm{~mm}$ de diâmetro e $300 \mathrm{~mm}$ de altura. Foram moldadas três amostras para cada mistura de concreto produzida.

As propriedades mecânicas do concreto resistência à compressão e resistência à tração por compressão diametral foram verificadas de acordo com as prescrições da NBR 5739 (ABNT, 2007) e da NBR 7222 (ABNT, 2011) respectivamente. Foram utilizados corpos de provas cilíndricos, com $100 \mathrm{~mm}$ de diâmetro e $200 \mathrm{~mm}$ de altura, para as idades 3, 7, 28 e 91 dias. Para cada dosagem foram moldados 12 corpos de prova a fim de serem ensaiados à compressão, e 12 corpos de prova para serem ensaiados à tração por compressão diametral, todos ensaiados no Laboratório de Materiais e Estruturas do UNASP.

\section{Resultados e discussões}

\section{Concreto no estado fresco}

Os dados que apontam as características dos concretos em análise no estado fresco são apresentados na Tabela 2 do CAAR e dos CAABCC.

Todos os concretos ensaiados apresentaram fluidez elevada. Mesmo assim, apresentaram grande coesão, sem qualquer indício de segregação. Na Figura 1 são apresentadas algumas fotos dos concretos no estado fresco evidenciando alta fluidez sem segregação, teor de argamassa adequado e bom aspecto de acabamento.

Observou-se, para as dosagens em análise, que os valores de fluidez (slump-flow test) atingiram o nível de autoadensabilidade especificado para as classes SF1 para o CAABCC3 e SF2 para os demais concretos, podendo, portanto, ser utilizados na fabricação de lajes, revestimento de túneis, estacas e certas fundações profundas para o SF1, e ser aplicados em paredes, vigas e pilares e outras para o SF2 (ABNT, 2017).

Os valores determinados para o ensaio de caixa-L, que indica a capacidade do concreto de fluir através do confinamento, classificam os concretos como sendo classe PJ1. Esta classe de concreto é propícia para uso em elementos estruturais com espaçamentos entre armaduras de $80 \mathrm{~mm}$ e $100 \mathrm{~mm}$, podendo ser aplicados em painéis, vigas, pilares, entre outros elementos estruturais, segundo a NBR 15823 (ABNT, 2017).

Visualmente, para o CAA ser considerado sem segregação no ensaio de espalhamento, o agregado deve estar homogeneamente distribuído na mistura e acompanhar a movimentação da argamassa até a extremidade do círculo formado pelo CAA. Na Figura 2 são apresentadas as bordas do espalhamento para cada concreto: CAAR, CAABCC1, CAABCC2 e CAABCC 3. 
Tabela 2 - Caracterização dos concretos de referência e de baixo consumo de cimento no estado fresco

\begin{tabular}{|c|c|c|c|c|c|}
\hline Propriedades & Limites NBR 15823 & CAAR & CAABCC1 & CAABCC2 & CAABCC3 \\
\hline Slump-flow, df (mm) & $550 \leq \mathrm{df} \leq 850$ & 680,00 & 697,50 & 750,00 & 605,00 \\
\hline T500 (s) & $>2$ & $5 ” 56$ & 4”91 & $08 ” 20$ & $10 ” 26$ \\
\hline Caixa L, h2/h1* & $\geq 0,80$ & 0,96 & 0,99 & 0,99 & 0,96 \\
\hline Caixa L, t20 (s) & -- & $1 ” 37$ & $1 ” 77$ & $2 " 68$ & $1 ” 56$ \\
\hline Caixa L, t40 (s) & --- & $3 ” 44$ & 3”07 & 7”13 & $5 ” 33$ \\
\hline Funil V, tv (s) & $9 \leq \mathrm{tv} \leq 25$ & 9”95 & 9”48 & $16 " 90$ & $16 " 65$ \\
\hline Tubo U & $\geq 0,90$ & 0,91 & 0,98 & 0,99 & 0,99 \\
\hline
\end{tabular}

Nota: *foram utilizados $4 \varphi 12,5 \mathrm{~mm}$ espaçados de $3,2 \mathrm{~cm}$.

Figura 1 - Aspectos dos concretos no estado fresco após o ensaio de espalhamento

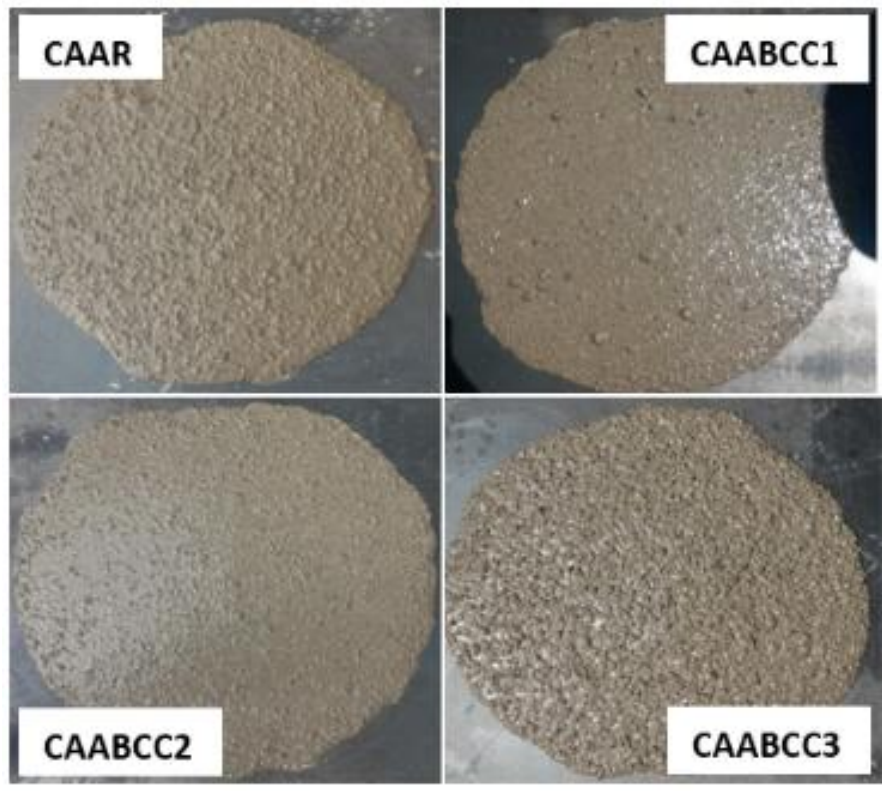

Figura 2 - Análise do aspecto visual do espalhamento dos concretos produzidos

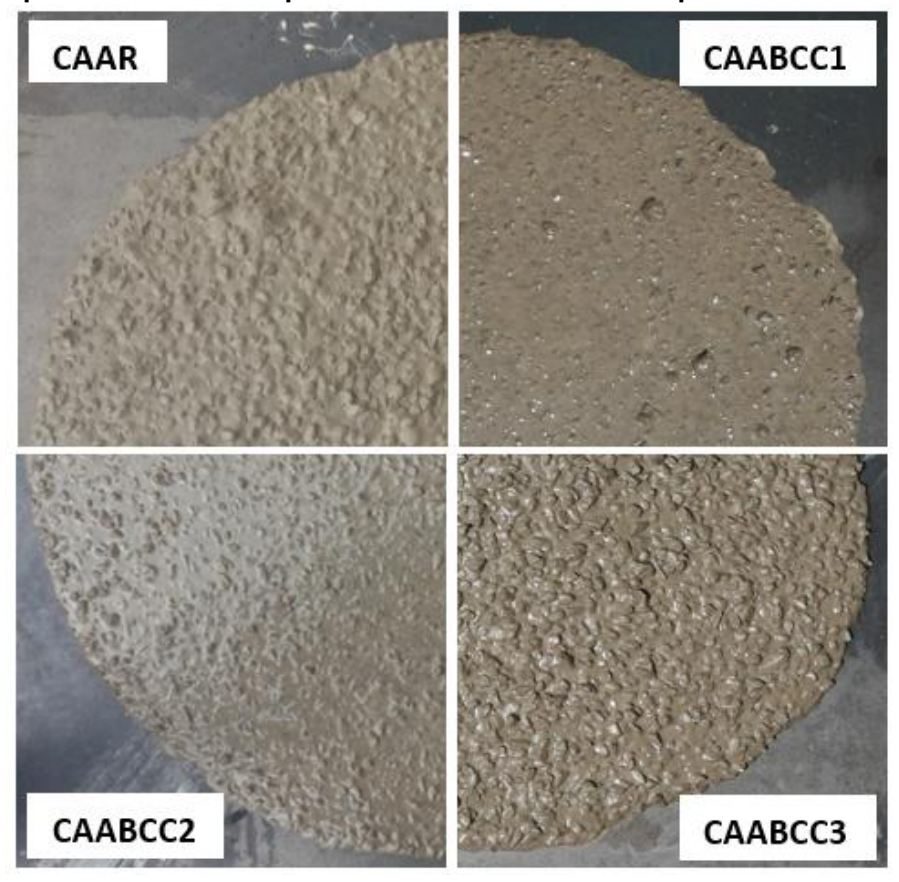

64 Barboza, L. da S.; Storch, I. S.; Almeida Filho, F. M. de 
Justifica-se o espalhamento do CAABCC3 ser o menor entre todos os concretos devido ao baixo volume de pasta e, consequentemente, de cimento. Portanto, decidiu-se não aumentar mais o consumo de água, pois as dosagens apresentavam indícios de início de exsudação. Esse é um problema que se encontra ao utilizarem consumos de cimento muito baixos. Nesse caso, os valores de espalhamento encontrados não significaram qualquer prejuízo em termos de trabalhabilidade ou acabamento, mas, caso fosse necessário maior abatimento, uma alternativa seria realizar ajustes nos demais finos presentes, possivelmente com consumo maior de sílica e/ou pó de calcário. Vale dizer que a ocorrência de exsudação é sempre indesejável, devendo ser evitada, pois proporciona perda de resistência à compressão.

\section{Concreto no estado endurecido}

São apresentados na Figura 3 os resultados obtidos para os ensaios de resistência à compressão realizados para os concretos.

Todas as dosagens de baixo consumo de cimento superaram a marca de $30 \mathrm{MPa}$ aos 28 dias, resistência considerada limite para a classe de agressividade II da NBR 12655 (ABNT, 2015).

De acordo com a Figura 3, pode-se observar a evolução do ganho de resistência à compressão dos quatro concretos estudados com o aumento das idades de cura, que pode ser devido ao aumento gradual do grau de hidratação do cimento (NGUYEN, 2019). Constata-se inicialmente melhor desempenho mecânico do concreto de referência (CAAR) na idade de 3 dias. Para os concretos com baixo consumo de cimento, observa-se que, com o avanço das idades, foram alcançadas resistências equivalentes ao CAAR a partir da idade de 7 dias para o concreto CAABCC2 e de 28 dias para os concretos CAABCC1 e CAABCC3. Segundo Neville (2016), quanto maior o consumo de cimento, maior será o aumento de resistência nas primeiras idades, o que retrata o comportamento do concreto de referência (CAAR).

Já o CAABCC1 praticamente não apresentou evolução de resistência após a idade de 28 dias. Ressalta-se que de 28 para 91 dias observaram-se ganhos proximamente de 4,19\%, 12,06\% e 12,10\% para CAABCC1, CAABCC2 e CAABC3 respectivamente. Esses ganhos são consideravelmente maiores que os previstos pela fórmula de previsão de ganhos de resistência do item 12.3.3 da NBR 6118 (ABNT, 2014). Esta indica para concretos com CPII ganho de apenas $11 \%$.

É possível verificar também que o CAABCC3, que apresentou o menor volume de pasta e, consequentemente, o menor consumo de cimento, teve resistência similar nas idades de 7, 28 e 91 dias em comparação ao concreto de referência. Acredita-se que esse comportamento é gerado pela maior compacidade conferida à pasta pelo aumento do consumo de areia muito fina na mistura. Pelisser, Vieira e Bernardin (2018) afirmam que misturas com maiores índices de empacotamento e misturas com diferentes distribuições granulométricas contribuem para a redução da porosidade da matriz de cimento e contribuem para o aumento da resistência à compressão.

Figura 3 - Resultados de resistência à compressão dos concretos em análise

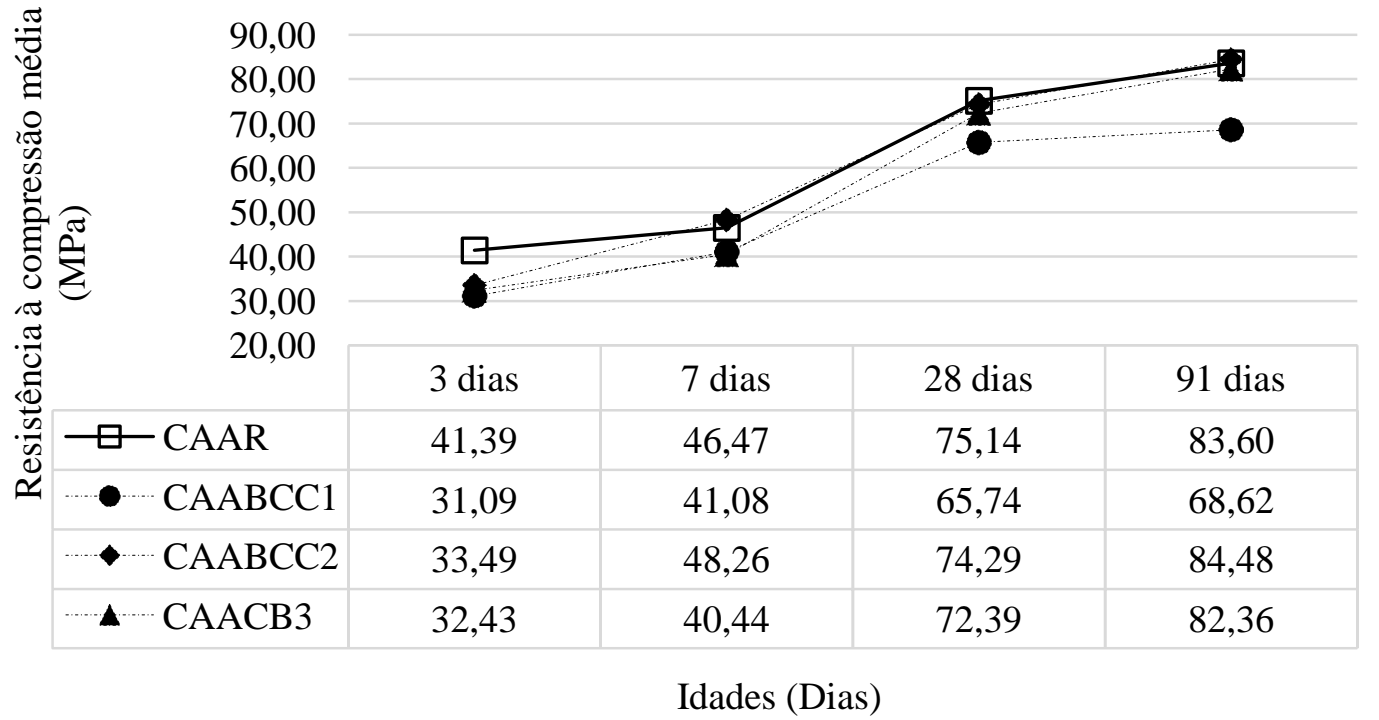


Segundo Okamura (1997), Domone (2006) e Zhao et al. (2015), quanto mais finas forem as partículas de agregado miúdo, maior a capacidade de fechar os poros não preenchidos totalmente com as partículas de agregado graúdo, o que aumenta, dessa forma, a densidade da mistura e, consequentemente, sua resistência à compressão. Além disso, há melhor hidratação das partículas de cimento por causa da maior retenção de água. Essa afirmação está de acordo com o que é apresentado por Repette (2005), a qual alega que tanto a hidratação do cimento quanto a aderência do CAA nas armaduras são melhoradas em função da maior retenção de água que esse tipo de mistura pode proporcionar.

Ainda, Nguyen (2019) afirmou que esqueletos granulares mais otimizados são cada vez mais necessários à medida que se reduz o consumo de cimento. O controle do empacotamento é fundamental para concretos com consumos reduzidos de cimento. Portanto, aspectos como tamanho dos grãos, distribuição granulométrica e morfologia dos agregados interferem na efetivação do empacotamento.

Na Figura 4 apresentam-se os resultados de resistência à tração por compressão diametral. Notam-se boas resistências dos concretos com baixo consumo de cimento. Observa-se também que os valores obtidos no ensaio de tração por compressão diametral para os concretos com baixo consumo de cimento se comportaram da mesma forma que o concreto de referência. Como todos apresentam aproximadamente a mesma resistência a tração, é possível concluir que a redução do cimento não comprometeu a resistência, nem positiva nem negativamente.

A massa específica do concreto no estado endurecido foi determinada no intuito de verificar se a redução do consumo de cimento interferiria em seu peso específico. Os concretos com baixo consumo de cimento possuem densidade média de aproximadamente $2,53 \mathrm{~g} / \mathrm{cm}^{3}$, e o de referência, $2,52 \mathrm{~g} / \mathrm{cm}^{3}$. Os resultados médios obtidos são apresentados na Tabela 3.

Verifica-se que a massa específica (Tabela 3) dos concretos com baixo consumo de cimento apresentaram-se mais densos que o concreto de referência. Esse aumento da densidade demonstra melhor empacotamento entre os grãos e substituição da pasta de cimento por agregados. Observa-se, no entanto, que as massas específicas obtidas são semelhantes às apresentadas por concretos convencionais, ou seja, não apresentam alteração significativa.

\section{Figura 4 - Resultados de resistência à tração por compressão diametral}

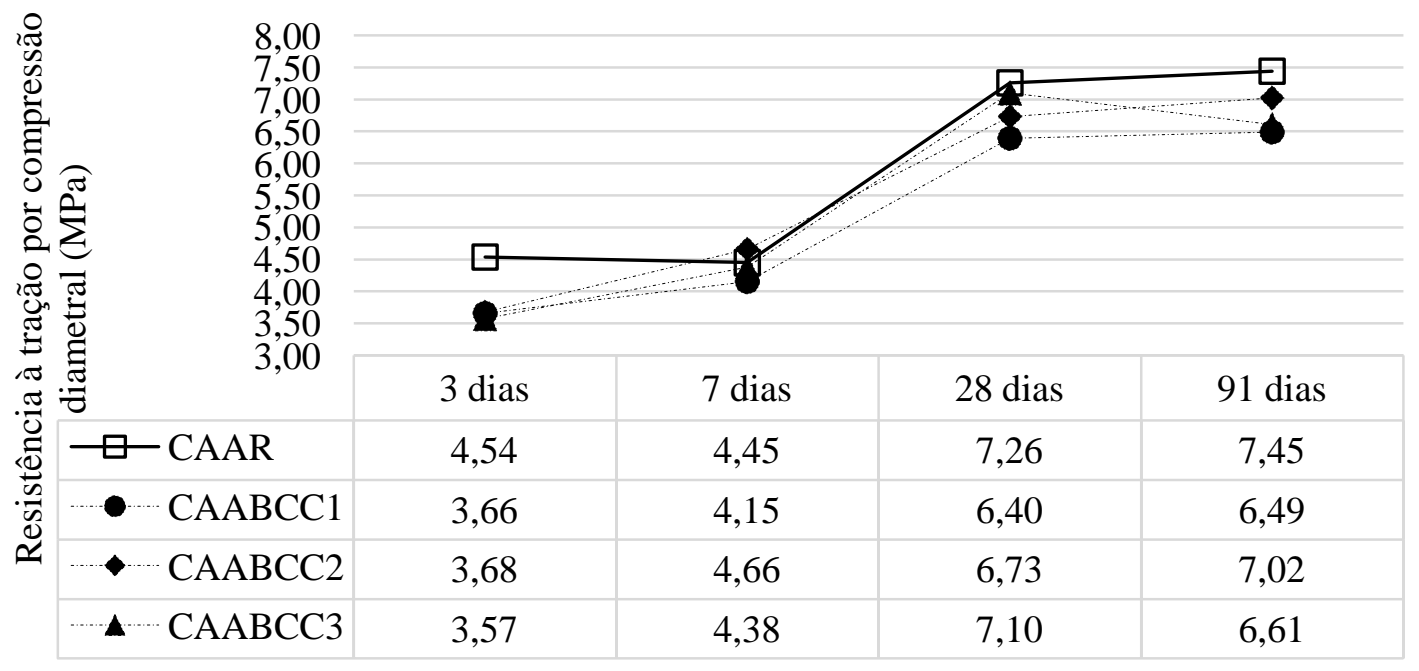

Idade (dias)

Tabela 3 - Massa específica no estado endurecido aos 28 dias

\begin{tabular}{c|c}
\hline Concretos & Massa específica $\left(\mathbf{g} / \mathbf{c m}^{\mathbf{3}}\right)$ \\
\hline CAAR & 2,52 \\
CAABCC1 & 2,57 \\
CAABCC2 & 2,55 \\
CAABCC 3 & 2,48 \\
\hline
\end{tabular}

66 Barboza, L. da S.; Storch, I. S.; Almeida Filho, F. M. de 


\section{Indicadores de consumo de cimento e emissão de $\mathrm{CO}_{2}$}

Geralmente a resistência à compressão é considerada como a propriedade mais importante do concreto, sendo quase que invariavelmente um elemento essencial do projeto estrutural. Mesmo no CAA, no qual as propriedades no estado fresco são tão importantes, o conhecimento da resistência à compressão se faz necessário para a comparação de seu custo de produção em relação a um concreto convencional. Desse modo, nesta pesquisa foi utilizada a resistência à compressão como parâmetro principal de avaliação de desempenho. Esse parâmetro foi avaliado em face do consumo de cimento, do consumo total de materiais cimentícios, do consumo de clínquer e da emissão aproximada de $\mathrm{CO}_{2}$.

$\mathrm{Na}$ Tabela 4 estão resumidos os consumos para volume de $1 \mathrm{~m}^{3}$ das diversas dosagens. O teor de clínquer no CP II Z 32 pode variar de $76 \%$ a $94 \%$ de acordo com as informações disponibilizadas pelo fabricante (VOTORANTIM, 2017). Portanto, adotou-se o valor mínimo de 76\%. De acordo com Pereira (2010), a emissão de $\mathrm{CO}_{2}$ para cimento CP II Z 32foi estimada em $0,84 \mathrm{~kg}$ de $\mathrm{CO}_{2}$ por quilo de clínquer.

Com basenesses consumos, e utilizando os valores de resistência à compressão obtidos nas idades analisadas, pode-se calcular os consumos relativos para as idades de 28 e 91dias (Tabela 5).

Notou-se em geral (Tabela 5) que os consumos relativos de cimento aos 28 dias ficaram entre $4,50 \mathrm{~kg} / \mathrm{m}^{3} \mathrm{e}$ $5,45 \mathrm{~kg} / \mathrm{m}^{3}$ por MPa para os concretos com baixo consumo de cimento, enquanto o concreto de referência, $5,78 \mathrm{~kg} / \mathrm{m}^{3}$ por $\mathrm{MPa}$.

Pelisser, Vieira e Bernardin (2018) produziram concretos autoadensáveis com consumo de cimento reduzido que atingiram em média um consumo de ligantes de $383,8 \mathrm{~kg} / \mathrm{m}^{3}$ para resistências de 20, 25, 30 e $40 \mathrm{MPa}$ aos 28 dias e obtiveram índices de 9,8, 9,1, 8,5 e 7,7 kg/m³/MPa respectivamente para os concretos. Em contrapartida, os resultados obtidos nesta pesquisa foram menores que os resultados apresentados por Pelisser, Vieira e Bernardin (2018), em que se obtiveram índices entre $4,50 \mathrm{~kg} / \mathrm{m}^{3}$ e $5,45 \mathrm{~kg} / \mathrm{m}^{3}$ por $\mathrm{MPa}$ para os concretos com consumo de cimento reduzido e de $5,78 \mathrm{~kg} / \mathrm{m}^{3}$ por MPa para o concreto de referência aos 28 dias, um consumo de cimento máximo para os CAABCC de $358,10 \mathrm{~kg} / \mathrm{m}^{3}$ e resistências superiores a $65 \mathrm{MPa}$ aos 28 dias, de forma que ficou comprovada a viabilidade dos concretos produzidos.

Firma-se mais uma vez que os concretos analisados se enquadram nos parâmetros usuais, em algumas circunstâncias apresentando comportamentos superiores e mais eficientes.

Realizando uma análise da emissão de $\mathrm{CO}_{2}$, as dosagens com baixo consumo de cimento CAABCC2 e CAABCC 3 mostraram ótimo desempenho, com valores abaixo de $3 \mathrm{~kg} / \mathrm{m}^{3}$ por $\mathrm{MPa}$.

De modo geral, os concretos em estudo apresentaram ótimo desempenho, tanto em termos de consumo de material cimentício como em termos de emissão de $\mathrm{CO}_{2}$, além do consumo total de cimento reduzido.

Tabela 4 - Massa específica no estado endurecido aos 28 dias

\begin{tabular}{c|c|c|c|c}
\hline \multirow{2}{*}{$\begin{array}{c}\text { Consumo } \\
\left(\mathbf{k g} / \mathbf{m}^{3}\right)\end{array}$} & \multicolumn{4}{|c}{ Dosagens } \\
\cline { 2 - 5 } CAAR & CAABCC1 & CAABCC2 & CAABCC3 \\
\hline Cimento & 434,06 & 358,10 & 347,25 & 325,54 \\
Cimentícios & 477,47 & 393,86 & 381,92 & 358,05 \\
Clínquer & 329,89 & 272,12 & 263,87 & 247,38 \\
CO $_{2}$ & 277,10 & 228,58 & 221,65 & 207,80 \\
\hline
\end{tabular}

Tabela 5 - Consumos relativos de cimento, material cimentício, clínquer e emissão de $\mathrm{CO}_{2}$ para as quatro dosagens analisadas quanto à resistência à compressão aos 28 e 91 dias $\left(\mathrm{kg} / \mathrm{m}^{3} / \mathrm{MPa}\right)$

\begin{tabular}{c|c|c|c|c|c}
\hline Idade & Consumo $\left(\mathbf{k g} / \mathbf{m}^{3}\right)$ & CAAR & CAABCC1 & CAABCC2 & CAABCC3 \\
\hline \multirow{4}{*}{ 28 dias } & Cimento & 5,78 & 5,45 & 4,67 & 4,50 \\
& Cimentícios & 6,35 & 5,99 & 5,14 & 4,95 \\
& Clínquer & 4,39 & 4,14 & 3,55 & 3,42 \\
& $\mathrm{CO}_{2}$ & 3,69 & 3,48 & 2,98 & 2,87 \\
\hline \multirow{4}{*}{91 dias } & Cimento & 5,19 & 5,22 & 4,11 & 3,95 \\
& Cimentícios & 5,71 & 5,74 & 4,52 & 4,35 \\
& Clínquer & 3,95 & 3,97 & 3,12 & 3,00 \\
& $\mathrm{CO}_{2}$ & 3,31 & 3,33 & 2,62 & 2,52 \\
\hline
\end{tabular}


Observou-se que foi possível atingir uma redução de $25 \%$ do consumo de cimento (CAABCC3) em comparação ao concreto de referência (CAAR). Como o CAAR apresentou um consumo de cimento de $434,06 \mathrm{~kg} / \mathrm{m}^{3}$, contra $325,54 \mathrm{~kg} / \mathrm{m}^{3}$ para o CAABCC3, houve uma redução de aproximadamente $110 \mathrm{~kg} / \mathrm{m}^{3}$.

\section{Análise da viabilidade econômica}

Para verificar a viabilidade econômica da elaboração de concretos dos tipos propostos neste trabalho, incluise aqui breve avaliação de custos. Essa verificação se limita ao custo de materiais, considerando que não sejam necessárias grandes alterações de custos na mão de obra e equipamentos para produção. Na Tabela 6 são apresentados os custos unitários dos diversos materiais utilizados. Tratou-se de valores aproximados praticados na região metropolitana de Campinas, cidade de Engenheiro Coelho, SP, para ano de 2017, segundo os fornecedores.

Na Tabela 7 estão calculados os custos por metro cúbico de cada uma das dosagens adquiridas. Na terceira linha dessa tabela, para a avaliação do custo-benefício, são apresentados os custos relativos, tomando-se como base a resistência à compressão média alcançada aos 28 dias por cada dosagem desenvolvida.

Constata-se que ocorreu uma diminuição de custo ao reduzir o consumo de cimento, redução de aproximadamente $6 \%$ quando comparados o concreto de referência e o CAABCC de menor custo, o que representa uma diferença de R \$27,79 por metro cúbico naquele ano. Conclui-se pela viabilidade econômica, uma vez que o custo-benefício é mais baixo em todos os concretos com baixo consumo de cimento.

Ressalta-se que a diferença de custo entre os concretos analisados não foi grande em relação ao preço do aditivo empregado. Nas dosagens com menor consumo de cimento, devido à redução da pasta, necessitou-se de um consumo maior de aditivo para que o concreto atingisse suas propriedades no estado fresco, por se tratar de um concreto autoadensável, o que aumentou o custo. É notório o impacto da redução do consumo de cimento e da emissão de $\mathrm{CO}_{2}$ para atingir $1 \mathrm{MPa}$ nos concretos com baixo consumo de cimento. Portanto, pode-se afirmar que a redução de custo pode ser bem mais expressiva em concretos que não exijam fluidez elevada, como é o caso do concreto autoadensável.

Deve-se lembrar que os valores aqui apresentados são apenas indicativos, podendo as conclusões variar com base no custo de cada material nas diversas regiões do Brasil. Também algumas variações nas propriedades dos materiais podem gerar um resultado de desempenho diferenciado. Assim sendo, para que seja possível reduzir o custo total dos concretos de baixo consumo, deve-se realizar estudos de otimização de consumo, principalmente dos itens mais caros, como fíler, adições minerais e aditivos. Alterações nas proporções desses materiais influenciam tanto o custo total como o desempenho, de modo que o estudo de otimização de custo se faz necessário para atingir menores custos totais e relativos. Por fim, para uma análise mais conclusiva e completa, seria preciso desenvolver estudo de campo para observar a viabilidade de produção, lançamento e aplicação desse concreto, a fim de obter dados mais completos de custos, incluindo os custos de equipamentos e da mão de obra para a produção e a aplicação do concreto.

Tabela 6 - Custos unitários dos materiais empregados $(\mathrm{R} \$ / \mathrm{kg})$

\begin{tabular}{c|c|c|c|c|c}
\hline $\begin{array}{c}\text { Cimento } \\
\text { CPII Z 32 }\end{array}$ & $\begin{array}{c}\text { Sílica } \\
\text { ativa }\end{array}$ & $\begin{array}{c}\text { Fíler } \\
\text { calcário }\end{array}$ & Brita 0 & $\begin{array}{c}\text { Areia } \\
\text { fina }\end{array}$ & Aditivo \\
\hline 0,40 & 1,15 & 0,75 & 0,064 & 0,058 & 7,00 \\
\hline
\end{tabular}

Tabela 7 - Custos unitários dos materiais empregados $(\mathrm{R} \$ / \mathrm{kg})$

\begin{tabular}{c|c|c|c|c}
\hline \multirow{2}{*}{ Parâmetros } & & & Dosagens & \\
\cline { 2 - 5 } & CAAR & CAABCC1 & CAABCC2 & CAABCC3 \\
\hline Custos materiais $\left(\mathbf{R} \$ \mathbf{m}^{\mathbf{3}}\right)$ & 514,43 & 508,14 & 505,56 & 486,64 \\
Resistência média 28 dias $\mathbf{( M P a )}$ & 75,14 & 65,74 & 74,29 & 72,39 \\
Custo relativo $(\mathbf{R} \$ \mathbf{m} / \mathbf{M P a})$ & 6,85 & 7,73 & 6,81 & 6,72 \\
\hline
\end{tabular}

68 Barboza, L. da S.; Storch, I. S.; Almeida Filho, F. M. de 


\section{Considerações finais}

(a) aplicando-se a metodologia de Gomes (2002), somando a redução do volume de pasta, conceitos de empacotamento e dispersão de partículas, foi possível desenvolver um concreto autoadensável com redução de 108,52 kg de cimento para $1 \mathrm{~m}^{3}$ de concreto, com resistência à compressão média aos 28 dias de 72,39 $\mathrm{MPa}$ e consumo de apenas $325,54 \mathrm{~kg}$ de cimento por $\mathrm{m}$ de concreto. Isso equivale a uma intensidade de ligantes de apenas $4,50 \mathrm{~kg} / \mathrm{m}^{3} / \mathrm{MPa}$, o que comprovadamente pode ser considerado baixo consumo de cimento para um concreto autoadensável. Nessa faixa de resistência são raras as citações técnicas e científicas de literaturas de concretos com intensidade de ligantes abaixo de $5,50 \mathrm{~kg} / \mathrm{m}^{3} / \mathrm{MPa}$ (REBMANN, 2011);

(b) todos os concretos ensaiados apresentaram fluidez elevada. Mesmo assim, apresentaram grande coesão, sem qualquer indício de segregação;

(c) todos os valores obtidos nos ensaios de estado fresco para os concretos em análise atenderam aos requisitos mínimos especificados pela NBR 15823 (ABNT, 2017). Portanto, podem ser classificados como concretos autoadensáveis;

(d) tratando da massa específica, notou-se que a redução do consumo de cimento, consequentemente menor volume de pastas, gerou aumento da densidade dos CAABCC. Tal comportamento é consequência da substituição da pasta de cimento menos densa por agregados mais densos;

(e) a redução do consumo de cimento não interferiu de maneira significativa nas propriedades mecânicas dos concretos, em algumas situações, inclusive, tendo apresentado comportamento bem próximo ao do concreto de referência. Especificamente, o CAABCC1 apresentou 12,50\% de resistência à compressão, maior valor entre todos os concretos estudados. Em contrapartida, o CAABCC2 e o CAABCC3 apresentaram $1,10 \%$ e 3,7\% respectivamente de redução de resistência à compressão;

(f) uma análise do desempenho alcançado por meio da correlação entre resistência mecânica e consumo de cimento, aglomerantes e clínquer mostrou que esses concretos atingiram excelentes resultados. $\mathrm{Na}$ resistência à compressão aos 28 dias, CAAR, CAABCC1, CAABCC2 e CAABCC3 atingiram respectivamente consumos relativos de $5,78 \mathrm{~kg} / \mathrm{m}^{3} / \mathrm{MPa}$ (equivalente a $6,35 \mathrm{~kg} / \mathrm{m}^{3} / \mathrm{MPa}$ de materiais cimentícios e $4,39 \mathrm{~kg} / \mathrm{m}^{3} / \mathrm{MPa}$ de clínquer), de $5,45 \mathrm{~kg} / \mathrm{m}^{3} / \mathrm{MPa}$ (equivalente a $5,99 \mathrm{~kg} / \mathrm{m}^{3} / \mathrm{MPa}$ de materiais cimentícios e 4,14 kg/m $3 / \mathrm{MPa}$ de clínquer), de $4,67 \mathrm{~kg} / \mathrm{m}^{3} / \mathrm{MPa}$ (equivalente a $5,14 \mathrm{~kg} / \mathrm{m}^{3} / \mathrm{MPa}$ de materiais cimentícios e 3,55 kg/m $3 / \mathrm{MPa}$ de clínquer) e, por fim, de $4,50 \mathrm{~kg} / \mathrm{m}^{3} / \mathrm{MPa}$ (equivalente a $4,95 \mathrm{~kg} / \mathrm{m}^{3} / \mathrm{MPa}$ de materiais cimentícios e $3,42 \mathrm{~kg} / \mathrm{m}^{3} / \mathrm{MPa}$ de clínquer). Valores de maior impacto podem ser obtidos aos 91 dias com o aumento da resistência à compressão;

(g) a avaliação aproximada de emissão de $\mathrm{CO}_{2}$ indica também o bom desempenho ambiental desse tipo de concreto, chegando a uma emissão de apenas $2,87 \mathrm{~kg} / \mathrm{m}^{3}$ por MPa para o CAABCC3, contra de 3,69 $\mathrm{kg} / \mathrm{m}^{3} / \mathrm{MPa}$ para o CAAR, valor aproximadamente $22,22 \%$ menor; e

(h) comparando os valores de resistência e eficiência obtidos pelos CAABCC com o CAAR, chega-se a uma redução de $25 \%$ de cimento da mistura.

\section{Referências}

ALI, M.; SAIDUR, R.; HOSSAIN, M. A review on emission analysis in cement industries. Renewable and Sustainable Energy Reviews, v. 15, n. 5, p. 2252-2261, Jun. 2011.

ALMEIDA FILHO, F. M. Contribuição ao estudo da aderência entre barras de aço e concretos autoadensáveis. São Carlos, 2006. 350 f. Tese (Doutorado em Engenharia de Estruturas) - Departamento de Engenharia Civil, Universidade de São Paulo, São Carlos, 2006.

ASSOCIAÇÃO BRASILEIRA DE NORMAS TÉCNICAS. NBR 12655: concreto de cimento Portland: preparo, controle e recebimento: procedimento. Rio de Janeiro, 2015.

ASSOCIAÇÃO BRASILEIRA DE NORMAS TÉCNICAS. NBR 13276: argamassa para assentamento e revestimento. Rio de Janeiro, 2002.

ASSOCIAÇÃO BRASILEIRA DE NORMAS TÉCNICAS. NBR 15823: concreto autoadensável. Rio de Janeiro, 2017.

ASSOCIAÇÃO BRASILEIRA DE NORMAS TÉCNICAS. NBR 5739: concreto: ensaio de compressão de corpos-de-prova cilíndricos. Rio de Janeiro, 2007. 
ASSOCIAÇÃO BRASILEIRA DE NORMAS TÉCNICAS. NBR 6118: projeto de estruturas de concreto: procedimento. Rio de Janeiro, 2014.

ASSOCIAÇÃO BRASILEIRA DE NORMAS TÉCNICAS. NBR 7222: concreto e argamassa: determinação da resistência à tração por compressão diametral de corpos de prova. Rio de Janeiro, 2011.

ASSOCIAÇÃO BRASILEIRA DE NORMAS TÉCNICAS. NBR 9778: argamassa e concreto endurecidos: determinação da massa específica, índice de vazios e absorção de água por imersão. Rio de Janeiro, 2009.

BARBOZA, L. S. Estudo sobre o impacto da redução de consumo de cimento na resistência à compressão do concreto autoadensável. São Carlos, 2016. 172 f. Dissertação (Mestrado em Estruturas e Construção Civil ) - Departamento de Engenharia Civil, Universidade Federal de São Carlos, São Carlos, 2016.

BARBOZA, L. S.; ALMEIDA FILHO, F. M. Concreto autoadensável com baixo consume de cimento: impacto da redução do consume de cimento na resistência à compressão. Revista Matéria, v. 23, n. 3, Sept. 2018.

BARBOZA, L. S.; ALMEIDA FILHO, F. M. Estudo sobre a redução do consume de cimento e emissão de $\mathrm{CO}_{2}$ para concreto de alto desempenho. Science and Engineering Journal, v. 24, n. 2, p. 131-142, jul./dez. 2015.

DOMONE, P. L. Self-compacting concrete: an analysis of 11 years of case studies. Cement and Concrete Composites, v. 28, n. 2, p. 197-208, Feb. 2006.

FELIX, E. F.; POSSAN, E. Balance emissions and $\mathrm{CO}_{2}$ uptake in concrete structures: simulation based on the cement content and type. IBRACON Structures and Materials Journal, v. 11, n. 7, p. 135-162, Feb. 2018.

GETTU, R.; AGUILLÓ, L. Estado del arte hormigón autocompactable y su caracterización (parte I). Cemento Hormigón, v. 861, p. 50-67, 2004.

GOMES, P. C. C. Optimization and characterization of high-strength selfcompacting concrete. Barcelona, 2002. 150 f. Tese (Doutorado em Engenharia Civil) - Departamento de Engenharia Civil, Universidade Politécnica de Catalunya, Barcelona, 2002.

GUNEYISI, E.; GESOGLU, M.; OZBAY, E. Strength and drying shrinkage properties of self-compacting concretes incorporating multi-system blended mineral admixtures. Constrution and Building Materials, v. 24, p. 1878-1887, 2010.

JAGADISH, V.; SUDHARSHAN, M. S.; RANGANATH, R. V. Experimental study for obtaining selfcompacting concrete. Indian Concrete Journal, v. 77, n. 8, p. 1221-1226, 2003.

LIMA, J. Avaliação das consequências da produção de concreto no Brasil para as mudanças climáticas. São Paulo, 2010. 151 f. Tese (Doutorado em Engenharia Civil) - Escola Politécnica, Universidade de São Paulo, São Paulo, 2010.

LONG, W.-J. et al. Sustainable design and ecological evaluation of low binder self-compacting concrete. Journal of Cleaner Production, v. 167, p. 317-325, 2017.

MADANDOUST, R.; MOUSAVI, Y. Fresh and hardened properties of self-compacting concrete containing metakaolin.Constrution and Building Materials, v. 35, p. 752-760, 2012.

MEHTA, P. K.; MONTEIRO, P. J. M. Concreto: microestrutura, propriedades e materiais. 2. ed. São Paulo: Ibracon, 2014.

NAJIM, K. B.; HALL, M. R. Mechanical and dynamic properties of self-compacting crumb rubber modified concrete. Construction and Building Materials, v. 27, n. 1, p. 521-530, Feb. 2012.

NEVILLE, A. M. Propriedades do concreto. 5. ed. Porto Alegre: Bookman, 2016.

NGUYEN, H.-A. Enhancement of engineering properties of slag-cement based self-compacting mortar with dolomite powder. Journal of Building Engineering, v. 24, p. 100738, 2019.

OKAMURA, H. Self-compacting high-performance concrete. Concrete Internacional, v. 19, n. 7, p. 50-54, July 1997.

PELISSER, F.; VIEIRA, A.; BERNARDIN, A. M. Efficient self-compacting concrete with low cement comsuption. Journal of Cleaner Production, v. 175, p. 324-332, Feb. 2018.

70 Barboza, L. da S.; Storch, I. S.; Almeida Filho, F. M. de 
PEREIRA, T. A. C. Concreto autoadensável, de alta resistência, com baixo consumo de cimento Portland e com adições de fibras de lã de rocha ou poliamida. São Carlos, 2010. $281 \mathrm{f}$. Dissertação (Mestrado em Engenharia de Estruturas) - Escola de Engenharia de São Carlos, Universidade de São Paulo, São Carlos, 2010.

REBMANN, M. S. Durabilidade de concretos estruturais com baixo consumo de cimento Portland e alta resistência. São Carlos, 2011. 211 f. Dissertação (Mestrado em Engenharia Civil) - Departamento de Estruturas, Escola de Engenharia de São Carlos, Universidade de São Paulo, São Carlos, 2011.

REPETTE, W. L. Concretos de última geração: presente e futuro. In: ISAIA, G. C.Concreto: ensino, pesquisa e realizações. São Paulo: Ibracon, 2005.

SANTOS, R. F. C. Estudo de concretos autoadensáveis, com aditivo incorporador de ar, utilizados na produção de paredes de concreto armado moldadas no local. São Paulo, 2012. $181 \mathrm{f}$. Dissertação (Mestrado em Habitação: Planejamento e Tecnologia) - Departamento de Tecnologia em construção de Edifícios, Instituto de Pesquisa Tecnológicas do Estado de São Paulo, São Paulo, 2012.

SINDICATO NACIONAL DA INDÚSTRIA DO CIMENTO. Relatório anual. Rio de Janeiro, 2010.

SINGH, N.; SINGH, S. P. Carbonation and electrical resistance of self-compacting concrete made with recycled concrete aggregates and metakaolin. Constrution and Building Materials, v. 121, p. 400-409, 2016.

SUKUMAR, B.; NAGAMANI, K.; RAGHAVAN, R. S. Evaluation of strength at early ages of selfcompacting concrete with high volume fly ash. Construction and Building Materials, v. 22, n. 7, p. 13941401, Jul. 2008.

VEJMELKOVA, E. et al. Properties of self-compacting concrete mixtures containing metakaolin and blast furnace slag. Construction and Building Materials, v. 25, p. 1325-1331, 2011.

VOTORANTIM. Cimento Portland composto (ficha técnica do produto). Disponível em: http://http://www.votorantimcimentos.com.br/hotsites/cimento/base.htm. Acesso em: 21 jun. 2017.

ZHAO, H. et al. The properties of the self-compacting concrete with fly ash and ground granulated blast furnace slag mineral admixtures. Journal of Cleaner Production, n. 95, p. 66-74, May 2015.

\title{
Agradecimentos
}

Os autores reconhecem o apoio financeiro do Centro Universitário Adventista de São Paulo (UNASP), para o desenvolvimento desta pesquisa, e das empresas Tecnosil e Grace,pelo fornecimento de materiais.

Lucas da Silva Barboza

Departamento de Engenharia Civil | Centro Universitário Adventista de São Paulo | Estrada Municipal Pastor Walter Boger, s/n, km 3,4 | Engenheiro Coelho - SP - Brasil | CEP 13448-900 | Tel.: (19)3858-9366 | E-mail: lucas.barboza@unasp.edu.br

Izabella Sant'anna Storch

Programa de Pós-graduação em Estruturas e Construcão Civil | Universidade Federal de São Carlos | Rodovia Washington Luiz, s/n | São Carlos - SP - Brasil | CEP 13565-905 | Tel.: (16) 3351-8111 | E-mail: izabella.storch@hotmail.com

Fernando Menezes de Almeida Filho

Programa de Pós-graduação em Estruturas e Construção Civil | Universidade Federal de São Carlos | E-mail: almeidafilho@ufscar.br

\author{
Ambiente Construído \\ Revista da Associação Nacional de Tecnologia do Ambiente Construído \\ Av. Osvaldo Aranha, $99-3^{\circ}$ andar, Centro \\ Porto Alegre - RS - Brasil \\ CEP 90035-190 \\ Telefone: +55 (51) 3308-4084 \\ Fax: +55 (51) 3308-4054 \\ www.seer.ufrgs.br/ambienteconstruido \\ E-mail: ambienteconstruido@ufrgs.br
}

